\title{
Multiple Regression Analysis of Diagnostic Predictors In Optic Nerve Disease
}

\author{
T.H. KIRKHAM, and S.G. COUPLAND
}

\begin{abstract}
SUMMARY: Demyelination is assumed to be the cause of the majority of cases of isolated optic neuritis. Because of the importance of establishing the presence of optic nerve dysfunction in patients suspected of having multiple sclerosis several new indices of optic nerve conduction have been reported including the visual evoked potential, the edge-light pupil cycle time, and the Pulfrich test. These measures purport to detect optic nerve dysfunction but with varying degrees of success. This study of 93 patients with clearly documented previous optic neuritis was conducted to determine the statistical relationship between these three measures and other clinical diagnostic indices for detection of previous optic nerve disease and
\end{abstract}

RÉSUMÉ: On estime que la démvélinisation est la cause de la plupart des névrites optiques isolées. Étant donné l'importance du diagnostic d'une dysfonction du nerf optique chez les malades qu'on croit atteints de sclérose en plaques, plusieurs nouveaux indices de conduction du nerf optique ont été signalés: le potentiel visuel évoqué, le cycle pupillaire (edge-light pupil (ycle time), et l'épreuve de Pulfrich. Ces mesures visent à dépister une atteinte du nerf optique mais les résultats qu'elles donnent sont variables. La présente étude porte sur 93 malades dont la névrité optique a fait l'objet d'un diagnostic précis et documenté. Elle a été effectuée pour déterminer la relation statistique entre ces trois modes d'évaluation et d'autres indices permettant de déceler une névrite optique the utility of the diagnostic predictors taken individually, and in combination. The other indices used were the presence of detectable optic atrophy, color vision defect and the presence of a relative afferent pupillary defect. The variables were submitted to linear stepwise multiple regression analysis which indicated that the presence of optic atrophy, defective color vision and prolonged pupil cycle time when used in combination provided the most useful diagnostic prediction of previous optic neuritis in this group of patients. The addition of visual evoked potentials, the Pulfrich test or presence of a relative afferent pupillary defect did not significantly increase predictive reliability.

antérieure ainsi que pour juger l'utilité de ces outils diagnostiques considérés individuellement ou ensemble. On a également eu recours à d'autres critères: constatation d'une atrophie optique, défaut de la vision des couleurs et présence d'un défaut pupillaire afférent. Nous avons étudié les variables selon une analyse progressive $\grave{a}$ régression multiple qui a révélé que l'atrophie du nerf optique, le défaut de vision des couleurs et un cycle pupillaire prolongé, utilisés ensemble, constituent le meilleur indice d'antécédents de névrité optique. L'adjonction de potentiels visuels évoqués, de l'épreuve de Pulfrich ou la présence d'un défaut pupillaire afférent relatif n'a pas amélioré la qualité des prédictions de façon significative.
From the McGill University Departments of Neurology, Neurosurgery and Ophthalmology and Montreal Neurological Hospital and Institute, Montreal, Québec. Canada.

Reprint requests to Dr. Trevor Kirkham, MD. Neuro-Ophthalmology Department, Room 201. Montreal Neurological Hospital, 3801 University St. Montreal Québec, Canada, H3A 2B4.

\section{INTRODUCTION}

Demyelination is generally assumed to be the cause of the majority of cases of isolated optic neuritis. Most attacks of optic neuritis are unilateral and although the patient may regain $6 / 6$ visual acuity and a good visual field, there usually remain subjective deficits of central visual function which do not recover. Such defects include color desaturation and reduction of brightness discrimination when viewing with the affected eye, transient lowering of visual acuity after exercise or elevation of body temperature (Uhthoff's symptom) and, occasionally, annoying symptoms from delayed optic nerve conduction giving rise to impaired depth discrimination (Pulfrich phenomenom). Many patients, however, do not recover fully and clinically detectable signs attest to the previous optic nerve disease including reduced visual acuity, color vision deficit, optic nerve atrophy and a relative afferent pupillary defect.

An episode of optic neuritis may be the herald symptom of multiple sclerosis or may occur at some time during the course of the disease. Published statistics on the relationship of optic neuritis to multiple sclerosis vary widely and the complexity of this issue has been recently discussed (Compston et al. 1978; Cohen et al. 1979; Paty and Ebers, 1979). Experience shows that many patients with multiple sclerosis who have no clear history of optic neuritis have ophthalmoscopic or other clinical evidence of optic nerve disease. Because of the frequency with which multiple sclerosis affects the visual pathways, even in the absence of overt optic neuritis, more sophisticated tests of visual function are being developed to aid in the diagnosis of this disease. Such tests include electrophysiological indices of 
optic nerve and visual pathway conduction as measured by visual evoked potentials, measurement of optic nerve conduction time as determined by the pupillary light reflex, the pupil cycle time, and perceptual appreciation of apparent depth changes in moving objects, the Pulfrich effect. While these various measures purport to detect optic nerve dysfunction with varying degrees of success, to date there has been no published data on the relative effectiveness of these tests. For this reason we studied a group of patients who had recovered from an attack of isolated optic neuritis, who had no other symptoms or signs to suggest multiple sclerosis, in order to determine the relative usefulness of the electrophysiological and clinical parameters in the detection of recovered optic nerve disease. We also wished to determine the statistical relationship between the various diagnostic indices for previous optic neuritis and to determine whether any single test or a composite set of examinations had greater predictive value of optic nerve dysfunction.

\section{MATERIAL AND METHODS}

\section{Patients}

We examined 93 patients who presented with a history and clinical findings of optic neuritis where demyelination was assumed to be the cause. All patients were initially seen within four weeks of the onset of symptoms, but the tests described in this study were not all necessarily performed within that period. Some patients were reexamined as much as 5 years later when all the tests described were performed. There was a history of previous optic neuritis involving the same or opposite eye in $57 \%$ of cases. This high incidence of bilateral or multipie attacks probably reflects the specialized referral nature of this neuroophthalmic department. The patients were aged between 17- 39 years (mean age, 24.6 years). None had hypertension, diabetes, syphilis, anemia or other systemic disease. None had symptoms or clinical manifestations of multiple sclerosis. All gave a history of an acute loss of vision which reached its lowest level within three days, remained stable for a few days and then improved. Many had mild ocular or periorbital pain, often worse on eye movement, which lasted up to a week and which either preceeded or commenced at the same time as the visual loss. No patient had disc swelling or hemorrhages or a field defect more compatible with ischemic optic neuropathy rather than demyelinating optic neuritis. None were treated with adreno cortico steroids since this had been convincingly shown to be of no value (Bowden et al. 1974; Gould et al. 1977). Visual acuities had recovered to $6 / 12$ or better and none had detectable visual field defects on kinetic Goldmann perimetry other than occasional constriction of the $I_{2}$ isopter.

\section{Clinical Examination}

Evaluation was made of visual acuity, color vision, pupillary reactions, pupil cycle time, visual fields on Goldmann perimeter, fundus examination after pupillary dilatation, intraocular pressure and the Pulfrich test.

Color vision was tested using the Ishihara plates. For the purpose of this study a color defect was defined as three or more incorrect responses with either eye in the absence of obvious errors from congenital achromatopsia in males. Hesitancy in reading the plates, while clinically useful, being subjective in nature was not recorded in this study.

Pupil cycle time was measured using a bright horizontal $0.5 \mathrm{~mm}$ beam on the Haag-Streit slit lamp in a darkened room. The patient fixated a near target with the eye not being tested. The slit beam was raised until it overlapped the inferior pupillary margin; the pupil reflexly constricted so that the iris blocked the beam, and the pupil then redilated allowing entry of the beam into the pupil once more. Fifty consecutive constriction-dilatation cycles were measured twice for each eye and timed using a hand held chronometer. The recorded times (to the nearest 0.5 second) for the two trials were averaged to derive the pupil cycle time for one constrictiondilatation cycle for each eye. In our laboratory we have established the normal range in adults aged $15-45$ years to be 807 \pm 43 msec. A pupil cycle time of over 900 msec. was considered to be abnormally delayed. Interocular pupil cycle time differences were not employed as diagnostic data in this study although normally a difference of $50 \mathrm{msec}$. would be considered abnormal.

The Pulfrich test was performed by swinging a red $15 \mathrm{~mm}$. bob on a steel wire positioned 4 meters from the patient. At this distance we have found normal observers to give more consistent responses in detecting apparent depth changes with neutral density filter attenuation than with closer distances. All patients were first given a trial to demonstrate the Pulfrich depth effect using a red glass in front of one eye. When convinced that the patient could indeed appreciate the Pulfrich effect the patient was asked to draw in the air with a finger any perceived elliptical pattern of the bob when presented to both eyes. A positive response was defined as the report of illusory depth changes in which the bob was seen swinging away from the affected eye.

The visual evoked potential was recorded over midline occiput $\left(0_{z}\right)$ and left and right occipital hemispheres $\left(0_{1}\right.$ and $\left.0_{2}\right)$ using $\mathrm{Ag}-\mathrm{AgCl}$ electrodes secured by collodion. All active sites were referred to the vertex $\left(\mathrm{C}_{2}\right)$. A Grass PS-2 photostimulator was positioned $56 \mathrm{~cm}$ from the patient to provide a 12 degree test field centered on a 60 degree surround. Surround luminance was maintained at $0.65 \mathrm{~cd} / \mathrm{m}^{2}$, while the test field was incrementally increased to $2.25 \mathrm{~cd} / \mathrm{m}^{2}$ by 128 single flashes presented at $1 \mathrm{flash} / \mathrm{second}$. The patient's pupils were dilated prior to examination. The visual evoked responses to 128 single flashes were amplified by Grass P5 amplifiers (Grass Instrument Co., Mass.) and signal averaged by a Nicolet Fabritek 1070 (Nicolet Instrument Co., Wisc.). Both eyes were recorded monocularly. The averaged-visual evoked potentials were plotted on an X-Y plotter and the peak latency of the major positive component was identified. Visual evoked potential delay was defined as the presence of a positive component peak latency value exceeding 3 standard deviations above the normal population mean latency.

The data were coded and statistically analysed using programs from the Statistical Package for Social Sciences (SPSS). Frequency distributions of each variable measured were generated. In addition, crosstabulation analysis of the pupil cycle time and visual evoked potential measures with every other variable were produced and significance tested. Finally, a correlation matrix of the entire variable set was derived and the intercorrelations tested for significance. The correlation matrix was then submitted to a multiple regression procedure which determined the set of diagnostic variables that, when combined, provided the best possible prediction of previous optic nerve demyelination. In addition the variables which did and did not contribute significantly to this prediction were determined.

\section{RESULTS}

Data was appropriately coded and statistically analyzed by several SPSS procedures. Frequency distributions of abnormal findings on all diagnostic variables are presented in Table I. A crosstabulation analysis was per- 
TABLE 1

Frequency Distribution of Abnormal Parameters in Optic Neuritis

\begin{tabular}{lc}
\hline \multicolumn{1}{c}{ Parameter } & Percentage Abnormal \\
\hline Presence of Optic Atrophy & $52 \%$ \\
Color Vision Abnormality & $53 \%$ \\
Pupil Cycle Time Delay & $74 \%$ \\
Visual Evoked Potential Delay & $40 \%$ \\
Relative Afferent Pupillary Defect & $39 \%$ \\
Pulfrich Test Positive & $26 \%$ \\
\hline
\end{tabular}

formed upon the frequency distributions to investigate the relationship of the two optic nerve conduction measures (pupil cycle time and visual evoked potential) to the other clinical findings.

Table 2 presents the pupil cycle time and visual evoked potential crosstabulation results. This contingency table comprises paired observations on two nominal variables (eg. pupil cycle time and color vision, pupil cycle time and relative afferent pupillary defect, etc.). Chi-square $\left(\chi_{2}\right)$ provides an appropriate test of the statistical independence or association between the two measures. The Yates correction for continuity (Ferguson, 1966) was applied in the estimation and significance testing of chi-square since numerous cells contained less than 10 individuals. Table 2 shows a significant relationship between pupil cycle time and fundus appearance $(\mathrm{p}<.01)$, visual evoked potentials $(p<.07)$, and relative afferent pupillary defect $(\mathrm{p}<$ .07 ). The presence of visual evoked potential delay appeared to be significantly associated with color vision deficits $(p<.05)$, presence of a relative afferent pupillary defect $(\mathrm{p}<.05)$, fundus pallor $(\mathrm{p}<.001)$ and pupil cycle time delay $(\mathrm{p}<.07)$ in this group of optic neuritis patients.

Table 3 presents a more extensive assessment of bivariate association within the set of diagnostic variables. Inspection of the intercorrelation matrix indicates that most bivariate correlations were significant.

After "dummy-coding" each individual's diagnostic group membership
(Cohen, 1968) a multiple linear regression analysis was performed so that the best possible prediction of previous optic neuritis was estimated from a weighed combination of the six predictor variables (presence of optic atrophy, color vision defect, pupil cycle time, visual evoked potential, presence of relative afferent pupil defect and Pulfrich test). The multiple correlation ( $R$ ) and weights were subjected to significance testing to determine which predictors did or did not contribute significantly to this prediction. These data (table 4) illustrate that while the stepwise addition of each predictor variable increases the multiple $-\mathrm{R}$ the increment in itself is only significantly increasing for the addition of the first three variables (presence of optic atrophy, color vision defect and pupil cycle time). After these variables had been entered into the linear prediction the multiple$R$ was not significantly increased by the addition of the results of the examination of visual evoked potentials, the presence of a relative afferent pupillary defect or the Pulfrich test (table 4).

\section{DISCUSSION}

Ninety three (93) patients with a well documented previous acute optic neuritis were examined and clinical and electrophysiological indices statis-

TABLE 2

Crosstabulation of PCT and VEP Findings with other Clinical Parameters

\begin{tabular}{|c|c|c|c|c|c|c|c|c|c|c|}
\hline & \multicolumn{2}{|c|}{ Optic Disc } & \multicolumn{2}{|c|}{ Color Vision } & \multicolumn{2}{|c|}{ RAPD } & \multicolumn{2}{|c|}{ VEP } & \multicolumn{2}{|c|}{ Pulfrich } \\
\hline & Normal & Atrophy & Normal & Abnormal & Absent & Present & Normal & Delayed & Positive & Negative \\
\hline PCT Normal (24) & 15 & 9 & 12 & 12 & 18 & 6 & 18 & 6 & 9 & 15 \\
\hline PCT Delayed (69) & 30 & 39 & 33 & 36 & 39 & 30 & 39 & 30 & 15 & 54 \\
\hline $\mathrm{X}^{2}$ & \multicolumn{2}{|c|}{16.94} & \multicolumn{2}{|r|}{.18} & \multicolumn{2}{|c|}{3.40} & \multicolumn{2}{|c|}{3.40} & \multicolumn{2}{|c|}{1.56} \\
\hline \multirow[t]{3}{*}{ Significance } & \multicolumn{2}{|c|}{$\mathrm{p}<0.1$} & \multicolumn{2}{|c|}{ n.s.: } & \multicolumn{2}{|c|}{$\mathrm{p}<.07$} & \multicolumn{2}{|c|}{$\mathrm{p}<.07$} & \multicolumn{2}{|c|}{ n.s. } \\
\hline & \multicolumn{2}{|c|}{ Optic Disc } & \multicolumn{2}{|c|}{ Color Vision } & \multicolumn{2}{|c|}{ RAPD } & \multicolumn{2}{|c|}{ PCT } & \multicolumn{2}{|c|}{ Pulfrich } \\
\hline & Normal & Atrophy & Normal & Abnormal & Absent & Present & Normal & Delayed & Positive & Negative \\
\hline VEP Normal (57) & 39 & 18 & 33 & 24 & 39 & 18 & 18 & 39 & 15 & 42 \\
\hline VEP Delayed (36) & 6 & 30 & 12 & 24 & 18 & 18 & 6 & 30 & 9 & 27 \\
\hline $\mathrm{X}^{2}$ & \multicolumn{2}{|c|}{25.75} & \multicolumn{2}{|c|}{6.36} & \multicolumn{2}{|c|}{3.98} & \multicolumn{2}{|c|}{3.40} & \multicolumn{2}{|c|}{0.01} \\
\hline Significance & \multicolumn{2}{|c|}{$\mathrm{p}<.001$} & \multicolumn{2}{|c|}{$\mathrm{p}<.05$} & \multicolumn{2}{|c|}{$\mathrm{p}<.05$} & \multicolumn{2}{|c|}{$\mathrm{p}<.07$} & \multicolumn{2}{|c|}{ n.s. } \\
\hline
\end{tabular}

PCT = pupil cycle time VEP = visual evoked potential $\mathrm{RAPD}=$ Relative afferent pupillary defect n.s. $=$ not significant 
TABLE 3

Intercorrelation Matrix of Diagnostic Variables

\begin{tabular}{llllll}
\hline & $\begin{array}{c}\text { Optic } \\
\text { Atrophy }\end{array}$ & $\begin{array}{c}\text { Color } \\
\text { Vision }\end{array}$ & PCT & VEP & RAPD \\
\hline Color Vision & $.500^{1}$ & & & & \\
PCT & $.277^{1}$ & $.160^{1}$ & & & \\
VER & .1252 & .1931 & -.102 & & \\
RAPD & $.420^{1}$ & .3791 & -.025 & .076 & \\
Pulfrich & $.231^{1}$ & .1591 & -.058 & -.032 & .5081 \\
\hline
\end{tabular}

$P C T=$ pupil cycle time $\quad V E P=$ visual evoked potential $\quad$ RAPD $=$ relative afferent pupillary defect Superscripts $1=$ $<.012=p<.05$

TABLE 4

Multiple Regression Analysis of Optic Neuritis

\begin{tabular}{lccccc}
\hline \multicolumn{1}{c}{$\begin{array}{c}\text { Variable } \\
\text { Entered }\end{array}$} & F to Enter & Significance & Multiple-R & Overall-F & $\begin{array}{c}\text { Overall } \\
\text { Significance }\end{array}$ \\
\hline $\begin{array}{l}\text { Optic Atrophy } \\
\text { Color Vision }\end{array}$ & 12.916 & .000 & .239 & 12.916 & .000 \\
$\quad \begin{array}{l}\text { Defect } \\
\text { Pupil Cycle Time }\end{array}$ & 3.588 & .060 & .270 & 8.329 & .000 \\
Pulfrich Test & 2.227 & .103 & .285 & 6.204 & .000 \\
VEP & 1.883 & .171 & .296 & 5.236 & .000 \\
RAPD & 0.350 & .555 & .310 & 3.697 & .002 \\
\hline
\end{tabular}

VEP $=$ Visual evoked potential $R A P D=$ Relative afferent pupillary defect

tically analyzed. The results indicated a better prediction of optic nerve disease when a number of tests were used rather than any single measure alone.

\section{Optic atrophy}

Pallor of the optic disc is a notoriously unreliable way of determining visual function. Following demyelination there is almost invariably some loss of axons which, in the anterior visual pathway, is usually reflected by optic disc atrophy. In more subtle cases there may be detectable loss of some of the retinal nerve fiber bundles when using red-free ophthalmoscopy. Frisén and Hoyt (1974) considered that slit like defects in the peripapillary nerve layer represented retrograde degeneration and loss of axon bundles produced by lesions in the anterior visual pathways and that such findings could exist in the absence of reduced visual acuity or color vision, or even without obvious disc pallor. This sign is usually easy to detect in darkly pigmented fundi and youth when the nerve fiber layer appears to be thicker than it is with increasing age, but we have found it difficult to detect in the absence of overt visual symptomatology. For the purposes of this study we confined ourselves to recognition of disc atrophy in one or both eyes with or without detection of nerve fiber bundle defects. Optic atrophy was detected in $52 \%$ of the patients. Subsequent linear stepwise regression analysis indicated that the presence of optic atrophy was the single most important predictor of optic nerve disease (table 4).

\section{Color vision deficits}

Acquired color vision defects are well recognised in optic nerve disease and the detection of a color vision difference between the two eyes or a deficit in a female patient is usually significant. Color vision deficits were found in 53\% (table 1) and proved to be a useful clinical index of optic nerve dysfunction. Multiple regression analysis revealed that color vision deficit was the second best single predictor of optic nerve disease when used in combination with fundus appearance (table 4).

\section{Pupil cycle time}

Miller and Thompson (1978) reported on pupil cycle time in patients with optic neuritis and found $79 \%$ of patients to be abnormal either on the basis of a prolongation of pupil cycle time or an abnormal interocular pupil cycle time difference. Our patient population was four times larger than theirs and we did not use interocular pupil cycle time difference as a reference in this study, but even so, found the pupil cycle time to be abnormal in $74 \%$ of our cases. The pupil cycle time measures the reflex arc of the pupil and depends on intact afferent and efferent pupillary innervation and the presence of normal iris musculature. In optic neuritis the delay is due to a defect in the afferent arc. In our patients more positives were yielded by this test than by any other clinical measure (table 1). Chisquare analysis of contingency table 2 indicated both pupil cycle time and visual evoked response delay were moderately related $(\mathrm{p}<.07)$. Miller and Thompson (1978) mentioned that one of their patients with abnormal pupil cycle time had also a delayed visual evoked potential. We found that both of these nerve conduction measures appear to be significantly related to clinical measures of optic nerve function including disc pallor and the presence of a relative afferent pupillary defect. It was also found that visual evoked potential delay was significantly related to abnormal color vision.

\section{Visual evoked potentials}

Visual evoked potential studies have become popular because of the high frequency of abnormalities detected in optic nerve conduction in populations of patients with definite multiple 
sclerosis. Heavy reliance is now being placed on visual evoked potential abnormalities in patients with suspected multiple sclerosis as a diagnostic aid. Many published series suggest that more reliance is being placed on the result of visual evoked potential studies rather than, or in the absence of, clinical evaluation of the visual system. Recent reviews are given by Trojaborg and Petersen, (1979); Tackmann et al. (1979); Neetens et al. (1979) and Wilson and Keyser, (1980). Visual evoked potentials were delayed in $40 \%$ of our patients (table 1). This represents a conservative estimate of visual evoked potential delay since we used 3 standard deviations above the mean as our delay criterion, a Z-score value which exceeds that used in previously mentioned studies of visual evoked potentials. Using these strict criteria we found that the test was not as sensitive as an index of optic nerve demyelination as the pupil cycle time (table 2). However, visual evoked potentials were found to be significantly related with the presence of optic atrophy. Wildberger and Van Lith (1976) showed in a small group of optic neuritis patients that in the late recovery period visual evoked potentials remained more affected than color vision. Interestingly, delayed visual evoked potential was not significantly related to a relative afferent pupillary defect (table 2, 3). Ellis (1974) showed delay in visual evoked potential latency to be unrelated to the magnitude of a relative afferent pupillary defect and stated that reduced amplitude of the visual evoked potential could be predicted from abnormal pupillary function. It should be recognised that visual evoked potentials, unlike relative afferent pupillary defects, optic atrophy and pupil cycle times, measure not only the anterior visual pathways but also the retrogeniculate visual pathways. Multiple sclerosis is well recognised to affect the white matter of the brain indiscriminantly but usually in a fairly symmetrical manner although retrogeniculate lesions do not often correlate well with visual symptoms (Adams, 1977). Thus, some of the delay measured in our patients could possibly be related to retrogeniculate disease since it is impossible to state categorically that none of them actually did not have other demyelinating foci despite the absence of clinical evidence for multiple sclerosis.

\section{Relative afferent pupillary defect}

A relative afferent pupillary defect is a reliable sign of optic nerve disease (Thompson, 1966) and is readily detected when examining the patient fixating at distance in a dim room using a bright flashlight. However, in cases where there is bilateral optic nerve disease the sign is often difficult to detect especially when the disease is fairly symmetrical between the two eyes. This might explain the low frequency of relative afferent pupillary defect detection (39\%) in our patients (table 1). Burde and Gullin (1975) found that even in patients who had well documented unilateral optic neuritis the relative afferent pupillary defect could only be detected in half of them when examined 6 months after the episode of optic neuritis. In our regression analysis, addition of the relative afferent pupillary defect variable did not significantly increase the overall predictive effectiveness for optic neuritis (table 4).

\section{Pulfrich test}

The Pulfrich test depends for its appreciation upon normal stereoscopic vision. Since some $30 \%$ of normal subjects do not have full stereoscopic vision (Richards, 1970) one would expect the Pulfrich test to be the least sensitive in unilateral optic nerve disease. We found the test only to be of value in patients whose vision was $6 / 12$ or better in the worst affected eye. Since it was often difficult for the patient to understand what was expected to be seen we decided to provide a practice trial using a redglass as a filter to ensure that the patient could appreciate the phenomenom and then test the patient without the red-glass. The Pulfrich test was the least frequently occurring predictor in our study occurring only in $26 \%$ of the patients. Obviously the Pulfrich test, like the relative afferent pupillary defect, is of less value if the patient has suffered bilateral, relatively symmetrical, disease so that the stimulus from both eyes is delayed equally. Further, there is evidence that prolonged viewing of a Pulfrich-like stimulus depresses the appreciation of motion in depth, the prevailing situation in patients with predominantly unilateral optic nerve disease (Regan and Beverley, 1973). Our findings after multiple linear regression analysis do not support the contention of Frisén et al. (1973) that the Pulfrich phenomenom is the most sensitive test to demonstrate optic nerve disease. Addition of this variable did not significantly increase the overall cumulative regression coefficient (table 4) and therefore did not contribute significantly to the prediction.

In summary, the techniques of multiple correlation have practical application in situations where it is necessary to combine a number of variables in such a way as to provide the best possible estimate of a criterion measure. We have described such a situation using six diagnositic predictors which were used to predict the presence of previous optic neuritis. The results of this stepwise regression procedure indicated that clinical predictors such as optic atrophy, defective color vision and the pupil cycle time when used in combination provide the most useful diagnostic prediction of previous optic neuritis. Other parameters such as delayed visual evoked potentials, presence of a relative afferent pupillary defect or a positive Pulfrich test do not significantly increase the clinicians predictive ability over the use of the previous three predictors. The pupil cycle time appears to be a more reliable predictor of optic nerve disease than the VER and adds considerably in diagnosing optic nerve disease. Complete neuroophthalmic examination including pupil cycle time should be done to increase the certainty of diagnosis of previous optic neuritis which may be of importance in the context of suspected multiple sclerosis. We are presently investigating a large group of patients with suspected multiple sclerosis to determine the value of such predictors of optic nerve dysfunction in aiding in the diagnosis of multiple sclerosis. 


\section{ACKNOWLEDGEMENTS}

Supported by grants from the Canadian National Institute for the Blind our of the E.A. Baker Foundation for the Prevention of Blindaess, the Town of Mount Royal Old Timers Hockey League, Kiwanis Club of Mount Royal and the St. Nicholas Church Mens' Club. Dr. Stuart G. Coupland is a Postdoctoral Fellow supported by the Multiple Sclerosis Society of Canada.

\section{REFERENCES}

ADAMS, C.W.M. (1977). Pathology of multiple sclerosis: progression of the lesion. Brit. Med. Bull., 33, 15-20.

BOWDEN, A.N., BOWDEN, P.M.A., FRIEDMANN, A.I, and ROSE, F.C. (1974). A trial of corticotrophin gelatin injection in acute optic neuritis. J. Neurol. Neurosurg. Psychiat., 37, 869-873.

BURDE, R.M. and GALLIN, P.F. (1975). Visual parameters associated with recovered retrobulbar optic neuritis. Amer. J. Ophthalmol., 79, 1034-1037.

COHEN, M.M., LESSELL, S. and WOLF, P.A. (1979). A prospective study of developing multiple sclerosis in uncomplicated optic neuritis. Neurology (Minn.), 29. 208-213.

COMPSTON, D.A.S., BATCHELOR, J.R.,
EARL, C.J. and McDONALD, W.I. (1978). Factors influencing the risk of multiple sclerosis developing in patients with optic neuritis. Brain, 101, 495-511.

ELLIS, C.J.K. (1979). The afferent pupillary defect in acute optic neuritis. J. Neurol. Neurosurg. Psychiat., 42, 1008-1017.

FERGUSON, G.A. (1966). Statistical analysis in psychology and education. McGraw-Hill Publishing Company. 2nd edition.

FRISEN, L. and HOYT, W.F. (1974). Insidious atrophy of retinal nerve fibres in multiple sclerosis. Arch. Ophthalmol., 82, 91-97.

FRISEN, L., HOYT, W.F., BIRD, A.C. and WEALE, R.A. (1973). Diagnostic uses of the Pulfrich phenomenom. Lancet, 2, 385.

GOULD, E.S., BIRD, A.C., LEAVER, P.K. and McDONALD, W.I. (1977). Treatment of optic neuritis by retrobulbar injection of triamcinolone. Brit. Med. J., 1, 1495-1497.

MILLER, S.D. and THOMPSON, H.S. (1978). Pupil cycle time in optic neuritis. Amer. $\mathrm{J}$. Ophthalmol., 85, 635-642.

NEETENS, A., HENDRATA, Y. and VAN ROMPAEY, J. (1979). Pattern and flash visual evoked responses in multiple sclerosis. J. Neurol., 220, 113-124.

PATY, D.W. and EBERS, G.C. (1979). Clinical prognostic markers in multiple sclerosis. Can. J. Neurol. Sci., 6, 382.
REGAN, D. and BEVERLEY, K.I. (1973). Disparity detectors in human depth per ception: evidence for directional selectivity. Science, 181, 877-879.

RICHARDS, W. (1970). Stereopsis and steroblindness. Exp. Brain Res., 10, 380-388.

TACKMANN, W., STRENGE, H., BARTH, R. and SOJKA-RAYTSCHEFF, A. (1979). Diagnostic validity for different components of pattern shift visual evoked potentials in multiple sclerosis. Eur. Neurol., 18 , 243-248.

THOMPSON, H.S. (1966). Afferent pupillary defects. Pupillary findings associated with defects of the afferent arm of the pupillary light reflex arm. Amer. J. Ophthalmol., 62, 860-873.

TROJABORG, W. and PETERSEN, E. (1979). Visual and somatosensory evoked cortical potentials in multiple sclerosis. J. Neurol. Neurosurg. Psychiat., 42, 323-330.

WILDBERGER, H.G.H. and VAN LITH, G.H.M. (1976). Color vision and visually evoked response (VECP) in the recovery period of optic neuritis. Mod. Probl. Ophthalmol., 17, 320-324.

WILSON, W.B. and KEYSER, R.B. (1980). Comparison of the pattern and diffuse-light visual evoked responses in definite multiple sclerosis. Arch. Neurol., 37, 30-34. 